\title{
EFECTOS A CORTO PLAZO DE LA CONTAMINACIÓN ATMOSFÉRICA SOBRE LA MORTALIDAD. RESULTADOS DEL PROYECTO EMECAM EN LA CIUDAD DE BARCELONA, 1991-95 *
}

\author{
Carme Saurina (1), María Antònia Barceló (1), Marc Sáez (1) y Aureli Tobias (2) \\ (1) Departament d'Economia. Universitat de Girona. \\ (2) Unitat de Recerca Respiratòria i Ambiental, IMIM. Barcelona. \\ $\left(^{*}\right)$ Este trabajo cuenta con una beca del Fondo de Investigaciones Sanitarias (Expediente núm. 97/0051-02).
}

\section{RESUMEN}

Fundamentos: La mayoría de los estudios que muestran la existencia de una asociación a corto plazo entre contaminación atmosférica y morbimortalidad, analizan los efectos de los contaminantes «clásicos» cuya fuente es la combustión. Sin embargo, los cambios en las fuentes de emisión. fundamentalmente hacia el tráfico rodado, han modificado la contaminación atmosférica, haciendo más importante los componentes foto-químicos, como el ozono $\left(\mathrm{O}_{3}\right)$ y el dióxido de nitrógeno $\left(\mathrm{NO}_{2}\right)$. Barcelona es una ciudad situada en una zona templada y su contaminación atmosférica proviene principalmente de las emisiones de los vehículos. El principal objetivo de este artículo es el de analizar la relación entre los contaminantes foto-químicos, $\mathrm{NO}_{2}$ y $\mathrm{O}_{3}$, y la mortalidad por diferentes causas, en la ciudad de Barcelona desde 1991 hasta 1995, utilizando el protocolo de análisis elaborado dentro del proyecto EMECAM.

Métodos: Variaciones diarias del número de muertes por todas las causas, del número de muertes por todas las causas entre mayores de 70 años, del número de muertes por causas del aparato circulatorio y por causas respiratorias se relacionan con las variaciones diarias de los contaminantes foto-químicos, a través de modelos de Poisson autoregresivos, controlando confusores como la temperatura, la humedad relativa, la estructura temporal sistemática y la estructura autoregresiva.

Resultados: Excepto la relación entre $\mathrm{O}_{3}$ y mortalidad a causa de enfermedades respiratorias, las relaciones entre contaminantes foto-químicos y mortalidad por todas las causas consideradas resultaron estadísticamente significativas. Los riesgos relativos de morir ante incrementos de $\mathrm{O}_{3}$ fueron mayores que ante incrementos de $\mathrm{NO}_{2}$, casi el triple entre enfermedades del aparato circulatorio. Los riesgos rclativos de morir por todas las causas son menores que por causas especificas y que para aquellos individuos mayores de 70 años. Los resultados del análisis por semestres son muy similares a los del global mostrando, en todo caso, riesgos relativos algo mayores en los meses cálidos (mayo a octubre).

Correspondencia:

Carme Saurina.

Departament d'Economia.

Universitat de Girona.

Campus de Montilivi.

17071 Girona.

Tel 972-41.87.36.

Fax 972-41.80.32.

Correo electrónico: csaurina(a)enterprise.udg.es

ABSTRACT

\section{The Short-term Impact of Air Pollution on the Mortality. Results of the $E M E C A M$ Project in the City of Barcelona, 1991-1995}

Background: Most of the studies which demonstrate the existence of a short-tcrm rclationship between air pollution and morbidity and the Mortality analyze the impact of «classic» pollutants which are by-products of combustion. Ilowever, the changes in the sources of these emissions, shifting basically toward road traffic, has made a change in air pollution, heightening the importance of the photochemical components, such as ozonc $\left(\mathrm{O}_{3}\right)$ and nitrogen dioxide $\left(\mathrm{NO}_{2}\right)$. Barcelona is a city located in a mild climate zone, and its air pollution comes mainly from vehicle emissions. The main objective of this article is that of analyzing the relationship between the photochemical pollutants, $\mathrm{NO}_{2}$ and $\mathrm{O}_{3}$ and the death rate for different causes in the city of Barcelona throughout the 1991-1995 pcriod, using the procedure for analysis set out as part of the EMECAM Project.

Methods: Daily changes in the number of deaths resulting from all causes, of the number of deaths for all causes of those over age 70 , of the number of deaths resulting from cardiovascular diseases, and of the number of deaths resulting from respiratory-related causes are related to the daily changes in the photochemical pollutants using autoregressive Poisson models, controlling confusion-causing variables such as the temperature, the relative humidity, the systematic time structure and the autoregressive structure.

Results: Except for the relationship between $\mathrm{O}_{3}$ and the mortality for causes involving respiratory diseases, the relationships between photochemical pollutants and the mortality for all the causes considered were statistically significant. The risks related to dying as a result of rises in $\mathrm{O}_{3}$ were greater than as a result of rises in $\mathrm{NO}_{2}$, almost triple among cardiovascular diseases. The risks related to dying for all the causes are lower than for specific causes and than for those individuals over age 70. The results of the analysis by six-month periods are quite similar to the overall results, revealing, in any event, relative risks somewhat greater during the warm months (May to October). 
Conclusiones: La contaminación fotoquímica, sobre todo la causada por $\mathrm{O}_{3}$, representa un riesgo para la salud. Fn el caso del $\mathrm{NO}_{2}$ podría ocurrir que no fuese más que un indicador de las partículas suspendidas o de otros contaminantes cuyo origen es el tráfico urbano. Podría existir cierta modificación entre semestres de los efectos del $\mathrm{O}_{3}$ sobre la mortalidad por causas del aparato circulatorio.

Palabras clave: Proyecto EMECAM., Mortalidad. Contaminación foto-química. Regresión de Poisson.
Conclusions: Photochemical pollution, especially that which is caused by $\mathrm{O}_{3}$, comprises a health risk. In the case of $\mathrm{NO}_{2}$, this might not be more than an indicators of the suspended particles or of other pollutants stemming from city traffic. There may be a certain adjustment between six-month periods of the impact of $\mathrm{O}_{3}$ on the mortality for causes of the circulatory system.

Key words: $E M E C A M$ Project. Mortality. Photochemical Pollution. Poisson Regression.

\section{INTRODUCCIÓN}

Diversos estudios epidemiológicos recientes han mostrado la existencia de una asociación a corto plazo entre aumentos en los niveles de contaminación atmosférica y efectos sobre la salud, incluso con niveles moderados de contaminación, a menudo por debajo de los estándares internacionales de calidad'. La mayoría de los estudios analizan los efectos de contaminantes «clásicos» cuya fuente es la combustión, tales como humos negros y dióxido de azufre. Sin embargo, los cambios en las fuentes de emisión, fundamentalmente los relativos al tráfico rodado, han modificado la contaminación atmosférica, haciendo más importante los componentes foto-químicos, como el ozono $\left(\mathrm{O}_{3}\right)$ y el dióxido de nitrógeno $\left(\mathrm{NO}_{2}\right)^{2}$.

Barcelona es una ciudad idónea para el estudio de los efectos de la contaminación atmosférica sobre la salud ${ }^{3}$. Situada en una zona templada, su contaminación atmosférica proviene, principalmente, de las emisiones de los vehículos. Los niveles de contaminación son elevados, aunque dentro de los estándares permitidos por las guías de calidad de la Organización Mundial de la Salud $^{4}$. Un análisis químico sobre la proporción de partículas en suspensión en el aire en la ciudad de Barcelona, realizado en $1993^{5}$, muestra que los gases emitidos por los vehículos son los causantes de un $35 \%$ del total de partículas obtenido, mientras que la actividad industrial y otras emisiones de calor (calefacciones) contribuyen únicamente a un $1 \%$. Cerca del $50 \%$ de las partículas encontradas en el análisis se asociaron a polvo ambiental. La correlación entre la temperatura y la contaminación es poco elevada, especialmente durante el verano $0^{6}$, lo que facilita el control de tal variable de confusión.

Se considera al ozono como uno de los contaminantes foto-químicos de mayor toxicidad. Diversos estudios, tanto de laboratorios como epidemiológicos ${ }^{2}$, sugieren importantes efectos de la exposición al $\mathrm{O}_{3}$, específicamente en cuanto a la reducción de la función pulmonar, aumento de síntomas respiratorios y no respiratorios, exacerbación del asma e incrementos en el número de admisiones y de urgencias hospitalarias. Por su parte, varios estudios epidemiológicos sugieren que los efectos a corto plazo del $\mathrm{NO}_{2}$ se manifiestan en molestias respiratorias, en hospitalizaciones y visitas a los servicios de urgencias por causas respiratorias y también como efectos sobre la función pulmonar aunque, en el caso del $\mathrm{NO}_{2}$, la evidencia no es del todo consistente.

Aunque limitada, la evidencia empírica sugiere efectos adversos sobre la mortalidad de los aumentos a corto plazo en los niveles dc $\mathrm{O}_{3}$ y de $\mathrm{NO}_{2}{ }^{2-3,7-10}$. El problema, sin embargo, es que es difícil separar tales efectos de los de otros contaminantes, en especial de las partículas.

Anteriores estudios realizados en Barcelona mostraron una estrecha relación entre los niveles de contaminación atmosférica y cl número de urgencias hospitalarias como consecuencia de la enfermedad pulmonar obstructiva crónica ${ }^{11}$ y del asma ${ }^{12}$. Sin embargo, los efectos del $\mathrm{NO}_{2}$ sólo parecieron evidentes en el caso del asma. Por contra, parece existir una relación entre contami- 
nantes foto-químicos y mortalidad total para mayores de 70 años y como consecuencia de enfermedades del aparato circulatorio ${ }^{3}$.

Parece ser, no obstante, que la relación se presenta en los meses de verano pero no durante los de invierno. Precisamente, la estacionalidad en la mortalidad y en los contaminantes foto-químicos, aspecto que abarca la mayor parte de la variabilidad de tales variables, presenta distintas fases (mientras el número máximo de muertos se suele producir en los meses de invierno, los niveles máximos de $\mathrm{O}_{3}$, por ejemplo, se producen en verano). Es por ello que deben utilizarse métodos adecuados para controlar tales problemas $^{13}$.

El principal objetivo de este artículo es el de analizar la relación entre los contaminantes foto-químicos, $\mathrm{NO}_{2}$ y $\mathrm{O}_{3}$, y la mortalidad por diferentes causas, en la ciudad de Barcelona, desde 1991 hasta 1995, utilizando el protocolo de análisis elaborado dentro del proyecto $E M E C A M$.

\section{MÉTODOS}

Los procedimientos seguidos en este estudio son los acordados en el protocolo $A P H E A$, ampliados y adaptados en el proyecto $E M E C A M^{14}$. El número diario de muertes, según las distintas causas, se relacionó con las variaciones diarias de los contaminantes ambientales, usando modelos de regresión de Poisson autoregresivos. Se utilizaron distintos indicadores para las causas de mortalidad analizadas y para los contaminantes atmosféricos contemplados, y se introdujeron variables para controlar posibles efectos confusores debidos a aspectos meteorológicos y temporales.

El número de muertes diarias ocurridas entre residentes en la ciudad de Barcelona durante el período 1991-1995, fue proporcionado por el Institut Municipal de la Salut del Ayuntamiento de Barcelona. Se consideró el número total de muertes debidas a todas las causas menos las causas externas
(CIE-9: 001-799), el número total de muertos mayores de 70 años (CIE-9: 001-799), número de muertes a causa de enfermedades circulatorias (CIE-9:390-459) y número de muertes por problemas respiratorios (CIE-9: 460-519). Por lo que respecta a los contaminantes foto-químicos analizados en este artículo, $\mathrm{NO}_{2} \mathrm{y} \mathrm{O}_{3}$, se utilizaron los valores máximos (en un día y en ocho horas respectivamente) obtenidos como promedio de las observaciones proporcionadas por tres estaciones automáticas. Cada estación representa un comportamiento distinto de contaminación atmosférica urbana: industrial, residencial y de tráfico elevado. Los métodos de determinación de los contaminantes fueron la quimioluminiscencia $\left(\mathrm{NO}_{2}\right)$ y la absorción ultravioleta $\left(\mathrm{O}_{3}\right)$. Los valores diarios de temperatura media y de humedad relativa se recogieron de una estación urbana perteneciente al área metropolitana de Barcelona. Los datos sobre contaminación y variables meteorológicas fueron suministrados por la Corporación Metropolitana de Barcelona.

La relación entre los contaminantes y la mortalidad se analizó utilizando modelos de regresión de Poisson autoregresiva, de manera independiente para cada contaminante y para cada una de las cuatro causas de muerte analizadas. En el análisis se siguieron tres etapas consecutivas ${ }^{14}$. Determinación de un modelo basal, utilizando una regresión de Poisson en la que la variable dependiente fue la transformada logarítmica del número de muertes y en el que se controlaron las variaciones temporales, las variaciones meteorológicas y otras variables confusoras. Se introdujeron los contaminantes y sucesivos retardos del contaminante de manera individual en cada uno de los modelos basales de la mortalidad por las diferentes causas. Finalmente, se estimaron modelos de Poisson autoregresivos (a través del método de máxima verosimilitud) para el «mejor» retardo (o valor corriente) del contaminante. Se consideró como «mcjor», cl modelo del retardo (o valor corriente) con menor deviance relativa (respecto a la deviance del modelo nulo). Las variables ex- 
plicativas fueron las seleccionadas en los dos pasos antcriores.

Con el fin de controlar el comportamiento de la tendencia a largo plazo, los modelos ajustados incluyeron un término lineal para la tendencia y una variable ficticia para cada año de los contemplados en el estudio, reservando 1991 como año base. La estacionalidad fue controlada introduciendo términos sinusoidales hasta el sexto orden. Se controlaron las variables de humedad y de temperatura introduciendo sus valores diarios de manera lineal y cuadrática. Se incluyeron variables ficticias para cada día de la scmana y otra para las fiestas que no cayesen en domingo. Los efectos de un posible periodo inusual, como las Olimpíadas, fueron controlados introduciendo una variable ficticia ( 1 entre el 15 de Julio hasta el 15 de Agosto de 1992, 0 en otro caso). Finalmente, se introdujo el número de casos de gripe, valores obtenidos semanalmente y usados como promedio diario. Se probó hasta el sexto retardo de los contaminantes, hasta el retardo siete de temperatura y de humedad y hasta el retardo quince para el número de casos de gripe. El modelo final de regresión de Poisson autoregresivo se ajustó incluyendo hasta el retardo seis de la variable dependiente. Con el fin de analizar si la relación de la contaminación con la mortalidad era diferente en los meses fríos (de noviembre a abril) y cálidos (de mayo a octubre) el análisis se repitió por semestres ${ }^{14}$. El software utilizado en el estudio ha sido los paquetes SPSS y EGRET.

\section{RESULTADOS}

La tabla 1 presenta la distribución y la variación estacional de los datos de mortalidad, contaminación y variables meteorológicas. Los diferentes indicadores de mortalidad presentan los valores máximos en invierno y los mínimos en verano $(p<0,005)$. $\mathrm{El} \mathrm{O}_{3}$ presenta valores elevados en primavera y verano $y$ valores mucho menores en otoño y en invierno $(\mathrm{p}<0,05)$. No se encontraron diferencias estadísticamente significativas en $\mathrm{NO}_{2}$ en las diferentes estaciones. $\mathrm{NO}_{2}$ y $_{3}$ estuvieron poco correlacionados en el periodo analizado $(r=0,295)$. Además, la correlación de estos contaminantes con otros más clásicos, tales como humos negros y dióxido de azufre, también fue reducida (correlación máxima de -0,334). La variable que presentó la correlación más alta con la temperatura fue $\mathrm{O}_{3}(\mathrm{r}=0,415)$ mientras que $\mathrm{NO}_{2}$ presentó la más baja $(-0,011)$.

En las figuras 1 y 2 se muestran, con un propósito meramente ilustrativo, los gráficos de dispersión y el ajuste no paramétrico de regresiones localmente ponderadas (lowess) de las relaciones crudas entre los contaminantes y la mortalidad por diferentes causas.

Tabla 1

Número diario de muertos, contaminantes $\left(\mu \mathrm{g} / \mathrm{m}^{3}\right)$, temperatura $\left({ }^{\circ} \mathrm{C}\right)$ y humedad relativa $(\%)$ en Barcelona por estaciones, durante el período 1991-1995

\begin{tabular}{|c|c|c|c|c|c|c|c|c|c|c|}
\hline & \multicolumn{2}{|c|}{ Todo el periodo } & \multicolumn{2}{|r|}{ Primavera } & \multicolumn{2}{|r|}{ Verano } & \multicolumn{2}{|r|}{ Otoño } & \multicolumn{2}{|r|}{ Invierno } \\
\hline & Media & Rango & Media & Rango & Media & Rango & Media & Rango & Media & Rango \\
\hline Mort. Total & 43,620 & $20-83$ & 41,533 & $24-68$ & 39,741 & $20-61$ & 42,754 & $22-76$ & 50,581 & $29-83$ \\
\hline Mort. $\geq 70$ & 31,044 & $12-61$ & 29,330 & $16-49$ & 27,904 & $13-49$ & 30,145 & $12-57$ & 36,882 & $20-61$ \\
\hline Circulatorias & 17,435 & $5-41$ & 16,578 & $5-36$ & 15,117 & $5-35$ & 16,738 & $6-35$ & 21,377 & 9.41 \\
\hline Respiratorias & 3,793 & $0-15$ & 3,561 & $0-11$ & 2,959 & $0-10$ & 3,510 & $0-13$ & 5,166 & $0-15$ \\
\hline Temperatura & 16,490 & $3,150-30,789$ & 16,210 & $7,890-24,721$ & 24,198 & $16,338-30,789$ & 14,757 & $6,991-24,396$ & 10,307 & $3,150-18,360$ \\
\hline Humedad & 71,521 & $34,458-79,667$ & 70,019 & $34,458-79,458$ & 71,403 & $37,521-78,500$ & 73,041 & $49,125-79,313$ & 71,757 & $51,813-79,667$ \\
\hline $\mathrm{NO}_{2}$ máx.horar & 91,042 & $15,500-388,000$ & 95,185 & $15,500-286,000$ & 88,842 & $23,000-323,000$ & 87,606 & $29,000-388,000$ & 92,67 & $43,000-296,000$ \\
\hline $\mathrm{O}_{3}$ máx. 8 horas & 67,529 & $0,000-236,000$ & 89,603 & $19,500-236,000$ & 83,991 & $29,000 \cdot 182,500$ & 42,300 & $0,000-161,000$ & 52,911 & $2,000-132,500$ \\
\hline
\end{tabular}


Figura 1

Gráficos de dispersión y ajuste localmente ponderado (lowess) de las relaciones entre contaminantes foto-químicos y mortalidad Barcelona, 1991-1995

Mortalidad total entre mayores de 70 años

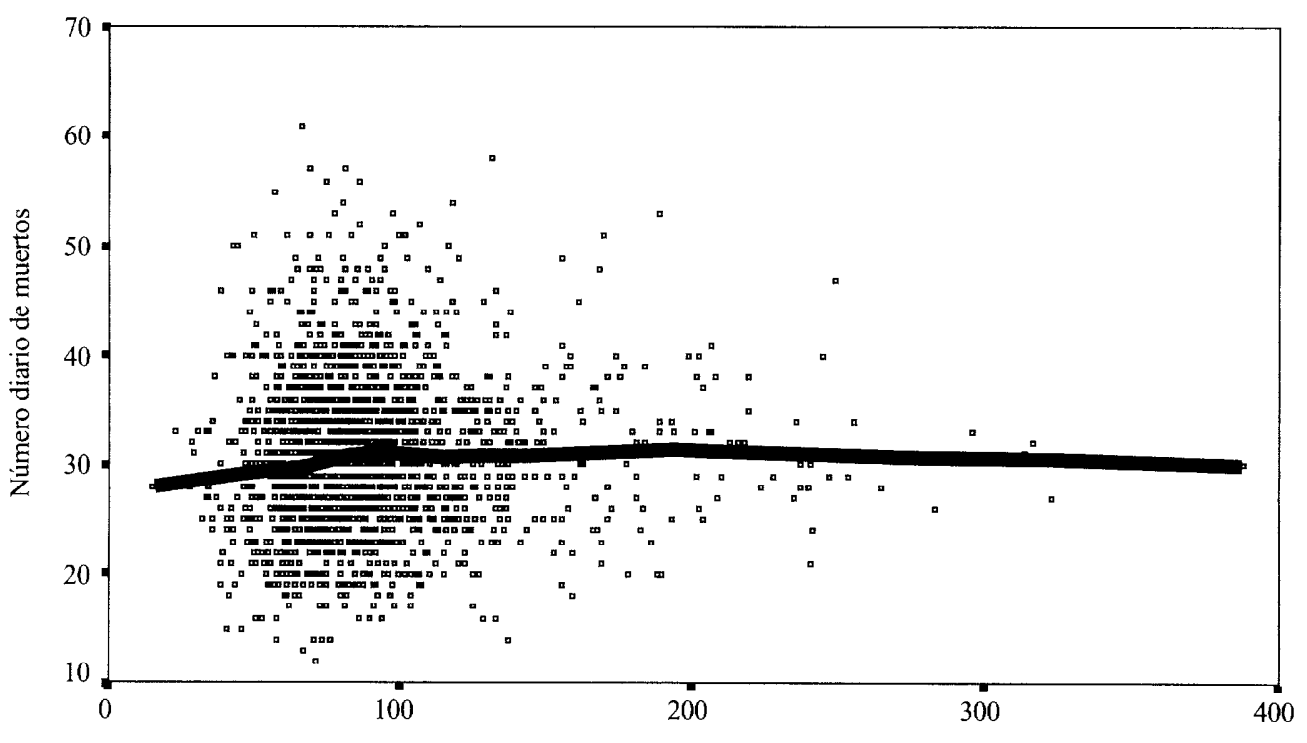

NO2 máxima horaria (en 24h)

Figura 2

Mortalidad consecuencia de enfermedades del aparato circulatorio

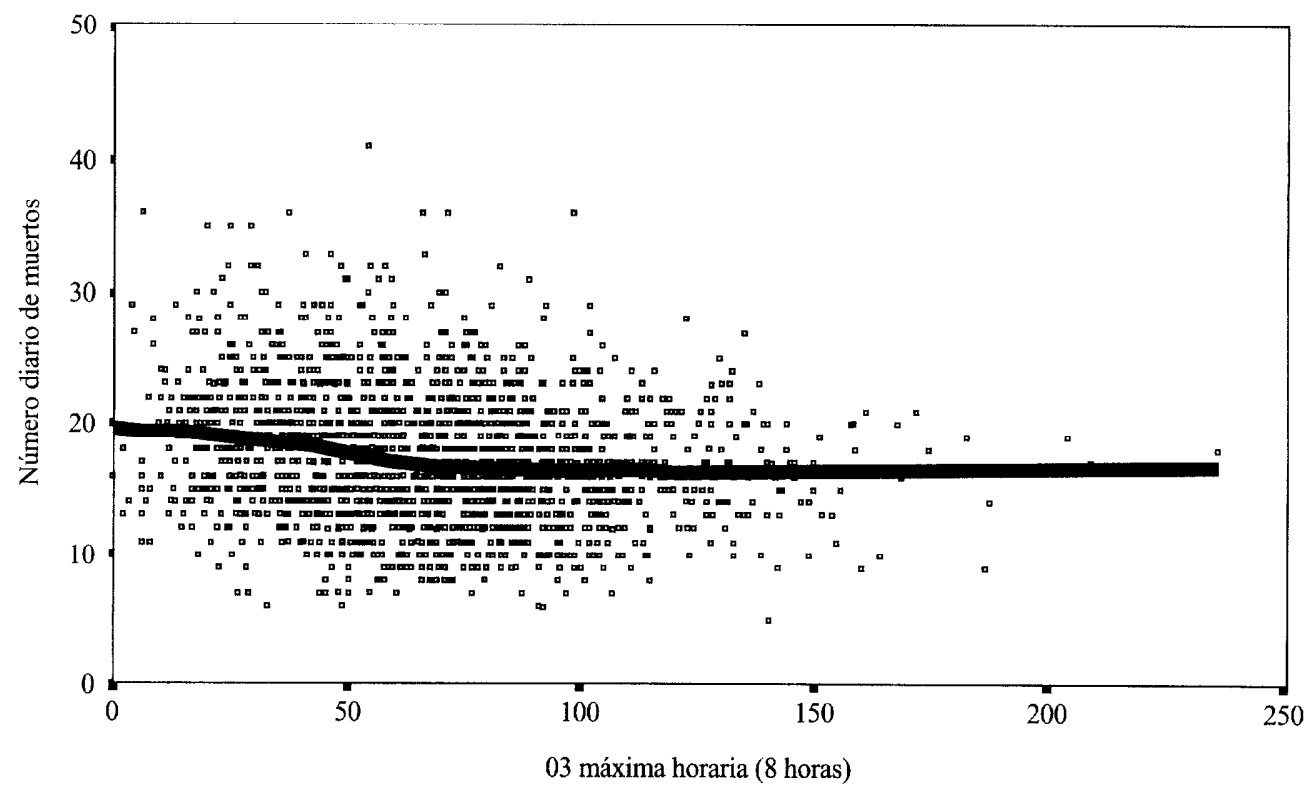


La tabla 2 muestra los riesgos relativos de morir por diferentes causas ante incrementos del contaminante de $10 \mu \mathrm{g} / \mathrm{m}^{3} \mathrm{y}$ ante aumentos equivalentes al rango intercuartílico del contaminante (Q3-Q1). Excepto la relación entre $\mathrm{O}_{3}$ y mortalidad a causa de enfermedades respiratorias, las relaciones entre contaminantes foto-químicos y mortalidad por todas las causas consideradas resultaron estadísticamente significativas $(p<0,05)$. El máximo riesgo relativo de morir se produce por causas del aparato circulatorio ante incrementos de $\mathrm{O}_{3}(1,03)$. Los riesgos relativos de morir ante incrementos de $\mathrm{O}_{3}$ (excepto el riesgo relativo en enfermedades respiratorias no estadísticamente significativo) fueron mayores que ante incrementos de $\mathrm{NO}_{2}$, particularmente entre enfermedades del aparato circulatorio $(1,0311$ vs 1,0114$)$. Las asociaciones se encontraron con el se- gundo retardo como máximo, excepto para $\mathrm{O}_{3}$ con la mortalidad respiratoria, las cuales presentaron asociaciones positivas (aunque no estadísticamente significativas) con el cuarto retardo. Los términos autorregresivos introducidos fueron hasta el sexto orden (en concreto el primero, el segundo y el sexto retardo de la variable dependiente). Aun así existió cierta sobre-dispersión, entre un 4\% (mortalidad total) hasta el 12\% (mortalidad por causa respiratoria), medida ésta como la fracción entre la deviance del modelo y los grados de libertad.

En la figura 3 se muestran los riesgos relativos de morir ante incrementos del contaminante de $10 \mu \mathrm{g} / \mathrm{m}^{3}$, estimados en el análisis por semestres. Excepto los riesgos correspondientes al $\mathrm{NO}_{2}$ en aparato circulatorio y causas respiratorias los riesgos relati-

Tabla 2

Riesgos relativos ajustados (intervalos de confianza al $95 \%$ ) de la mortalidad por cada $10 \mu \mathrm{g} / \mathrm{m}^{3}$ y por el rango intercuartílico, utilizando modelos de Poisson autoregresivos

$10 \mu \mathrm{g} / \mathrm{m}^{3}$

\begin{tabular}{|lcccc|}
\hline & Total & Total $\geq 70$ años & Circulatorio & Respiratorio \\
\hline $\mathrm{NO}_{2}$ & $1,00250(\mathrm{p}=0,017)$ & $1,00288(\mathrm{p}-0,023)$ & $1,00341(\mathrm{p}=0,043)$ & $1,00803(\mathrm{p}=0,016)$ \\
& $(1,00045-1,00454)$ & $(1,00039-1,00538)$ & $(1,00011-1,00672)$ & $(1,00168-1,01441)$ \\
Máx. horaria & Retardo: $\mathrm{t}-1$ & Retardo: $\mathrm{t}-1$ & Retardo: $\mathrm{t}-2$ & Retardo: $\mathrm{t}$ \\
\hline O3 & $1,00353(\mathrm{p}=0,034)$ & $1,00392(\mathrm{p}=0,044)$ & $1,00714(\mathrm{p}=0,007)$ & $1,00505(\mathrm{p}=0,349)$ \\
& $(1,00027-1,00679)$ & $(1,00010-1,00774)$ & $(1,00196-1,01235)$ & $(0,99451-1,01571)$ \\
Máx. 8 horas & Retardo: $\mathrm{t}-1$ & Retardo: $\mathrm{t}$ & Retardo: $\mathrm{t}-1$ & Retardo: $\mathrm{t}-4$ \\
\hline
\end{tabular}

Ajustando tendencia, términos sinusoidales, dia de la semana, festivos, temperatura, humedad, días inusuales, gripe, autocorrelación.

Rango intercuartílico (Q3-Q1)

\begin{tabular}{|lcccc|}
\hline & Total & Total $\geq 70$ años & Circulatorio & Respiratorio \\
\hline NO2 & $\begin{array}{l}1,00833(\mathrm{p}=0,017) \\
(1,00152-1,01588)\end{array}$ & $\begin{array}{l}1,00962(\mathrm{p}=0,023) \\
(1,00130-1,01801)\end{array}$ & $\begin{array}{l}1,01139(\mathrm{p}=0,043) \\
(1,00037-1,02252)\end{array}$ & $\begin{array}{l}1,02695(\mathrm{p}=0,016) \\
(1,00560-1,04872)\end{array}$ \\
$\mathrm{Q} 3-\mathrm{Q} 1=33,25$ & & & & \\
Máx. horaria & Retardo: $\mathrm{t}-1$ & Retardo: $\mathrm{t}-1$ & Retardo: $\mathrm{t}-2$ & Retardo: $\mathrm{t}$ \\
\hline O3 & $1,01526(\mathrm{p}=0,034)$ & $1,01695(\mathrm{p}=0,044)$ & $1,03109(\mathrm{p}=0,007)$ & $1,02191(\mathrm{p}=0,349)$ \\
& $(1,00115-1,00295)$ & $(1,00041-1,03371)$ & $(1,00848-1,05419)$ & $(0,97661-1,06931)$ \\
Q3 - Q1 =43 & Retardo: $\mathrm{t}-1$ & Retardo: $\mathrm{t}$ & Retardo: $\mathrm{t}-1$ & Retardo: $\mathrm{t}-4$ \\
\hline
\end{tabular}

Ajustando tendencia, términos sinusoidales, día de la semana, festivos, temperatura, bumedad, dias inusuales, gripe, autocorrelación. 
Figura 3

Riesgos relativos ajustados (intervalos de confianza al $95 \%$ ) de la mortalidad por cada $10 \mu \mathrm{g} / \mathrm{m}^{3}$ utilizando modelos de Poisson autoregresivos. Análisis global y por semestres
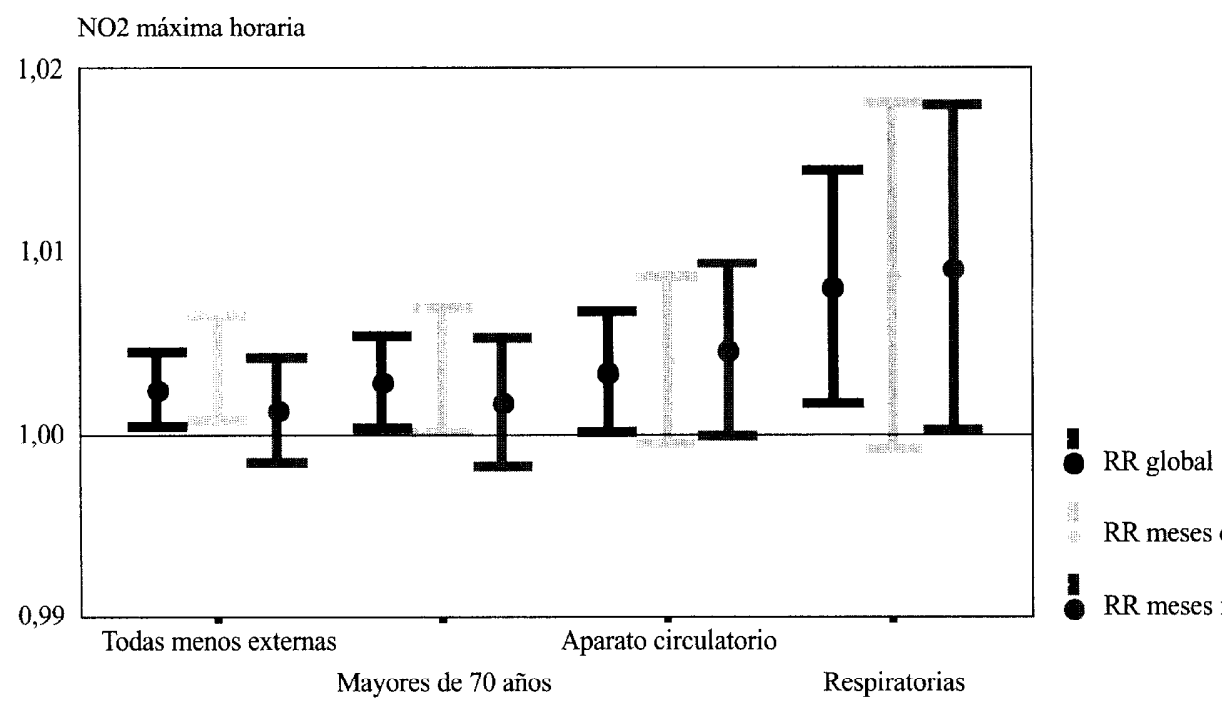

RR meses cálidos

CAUSA

O3 máxima 8 horas

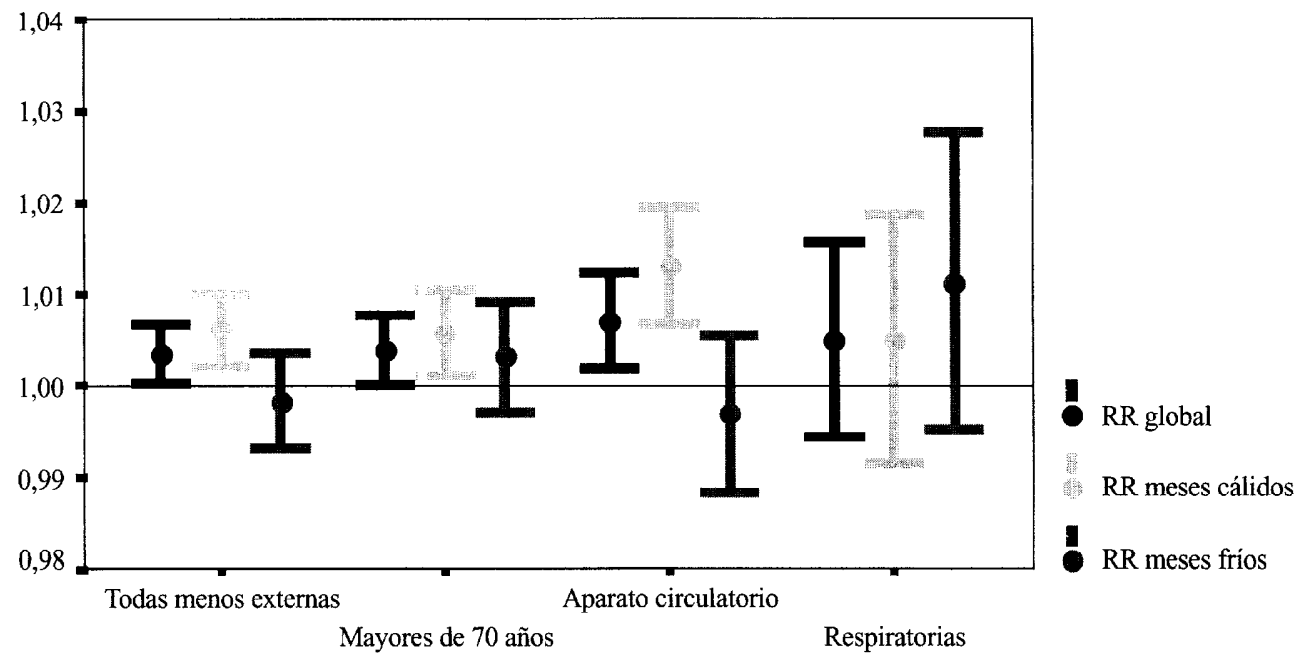

CAUSA

vos de los meses fríos (noviembre a abril) no son estadísticamente significativos, mientras que los correspondientes a los meses cálidos (mayo a octubre), excepto por lo que hace a causas respiratorias, resultaron estadísticamente significativos. Los riesgos relativos obtenidos en el análisis por semestres son, en general, mayores que los riesgos rclativos del análisis global, aunque sólo son estadísticamente diferentes (si bien con 
$\mathrm{p}<0,1)$ en el caso del riesgo relativo de morir por causas del aparato circulatorio ante incrementos de $\mathrm{O}_{3}$ en meses cálidos (mayo a octubre).

Excepto la relación entre $\mathrm{O}_{3}$ y mortalidad a causa de enfermedades respiratorias, las relaciones entre contaminantes foto-químicos y mortalidad por todas las causas consideradas, resultaron estadísticamente significativas. Los riesgos relativos de morir ante incrementos de $\mathrm{O}_{3}$ (excepto el riesgo relativo en enfermedades respiratorias, no estadísticamente significativo) fueron mayores que ante incrementos de $\mathrm{NO}_{2}$, casi el triple entre enfermedades del aparato circulatorio. Consecuencia quizás de su generalidad, los riesgos relativos de morir por todas las causas son menores que por causas específicas y mayores que los riesgos relativos de morir para aquellos individuos con más de 70 años. Los resultados del análisis por semestres son muy similares a los del global mostrando, en todo caso, riesgos relativos algo mayores en los meses cálidos (mayo a octubre).

\section{DISCUSIÓN}

Pocos estudios han investigado específicamente la relación a corto plazo entre la contaminación foto-química y mortalidad. Kinney y Ozkaynak ${ }^{7}$ encuentran asociaciones positivas entre $\mathrm{NO}_{2}, \mathrm{O}_{3}$ y la mortalidad total y por causa cardiovascular en el condado de Los Ángeles de Estados Unidos. No encontraron una relación significativa para la mortalidad por causas respiratorias, atribuyéndolo al reducido número de muertos por dicha causa. Similares resultados, incluso por lo que se refiere a la falta de significación para la mortalidad respiratoria, fueron encontrados para Barcelona, 1985-1991 por Sunyer et al. ${ }^{3}$. Touloumi ${ }^{15}$ también encuentra relaciones positivas significativas entre $\mathrm{NO}_{2} \mathrm{y} \mathrm{O}_{3} \mathrm{y}$ el número diario de muertos por todas las causas (excepto externas) en Atenas. Ostro et al. ${ }^{10}$, en un análisis para Santiago de Chile, sugieren que $\mathrm{O}_{3}$ puede tener un efecto independiente de otros contaminan- tes, partículas en especial, sobre la mortalidad total en verano. Estos mismos autores encuentran una asociación entre $\mathrm{NO}_{2}$ y mortalidad total, pero no parece que ésta sea independiente, por cuanto disminuye tras introducir en el modelo la contaminación por partículas (en concreto $\mathrm{PM}_{10}$ ). En este artículo hemos encontrado asociaciones en el mismo sentido que las descritas. Nótese que Barcelona y las ciudades citadas comparten clima, condiciones geográficas, fuentes y niveles de contaminación similares.

En el proyecto $A P H E A^{2}$ se encontraron efectos adversos del $\mathrm{O}_{3}$ sobre el número diario de muertos por todas las causas (excepto externas) en todas las ciudades estudiadas, aunque éstos difieren sustancialmente entre sí, por lo que respecta a condiciones geográficas, socio-demográficas y en calidad ambiental (niveles y composición de la contaminación atmosférica). El riesgo relativo de morir como consecuencia de un incremento de $50 \mu \mathrm{g} / \mathrm{m}^{3}$ en los niveles de ozono se estimó dentro de un rango de 1,3 a $8,6 \%$ y $2,9 \%$ fue el riesgo combinado $\left(\mathrm{IC}_{95 \%}, 1,0-4,9\right)$. Compárese con el riesgo estimado en este artículo, 1,76\% ( $\left.\mathrm{IC}_{95 \%}, 0,14-3,39 \%\right)$. Los resultados del meta-análisis del proyecto APHEA muestran una asociación importante para la mortalidad por causa circulatoria (al igual que nosotros) y una significación sólo marginal para la mortalidad por causas respiratorias (en nuestro caso no resultó significativa). No se conoce el mecanismo biológico que vincula el $\mathrm{O}_{3}$ con la mortalidad. Es posible, sin embargo, que los efectos adversos del mismo sobre la función pulmonar puedan suponer una amenaza para la vida, en particular para aquellos individuos con una enfermedad pulmonar crónica ${ }^{7}$.

Aunque globalmente el proyecto $A P H E A$ encuentra efectos adversos (marginalmente significativos) de un incremento de $50 \mu \mathrm{g} / \mathrm{m}^{3}$ en los niveles $\mathrm{NO}_{2}$ sobre la mortalidad total $(\mathrm{RR}=1,3 \%$, IC $95 \%=0,9-1,8)$, el rango de riesgos es tan amplio $(0,5-2,7)$ y la significación estadística tan escasa (únicamente en la mitad de las ciudades del proyecto APHEA 
lo es marginalmente) que nos impide compararlo con los hallazgos de este artículo. Nuestros resultados, como era de esperar, están en la línea de los encontrados para Barcelona en otros periodos temporales ${ }^{3,12}$.

Con el $\mathrm{NO}_{2}$, podría ocurrir que no fuese más que un indicador de las partículas suspendidas o de otros contaminantes cuyo origen es el tráfico urbano. En cualquier caso, la independencia de los efectos de los contaminantes foto-químicos con respecto a otros contaminantes necesita investigación adicional.

Desde el punto de vista estadístico, los modelos han sido razonablemente ajustados cumpliendo todos los instrumentos de diagnóstico. Así, por ejemplo, el modelo de Poisson autoregresivo es estacionario, por cuanto la suma de los estimadores de los parámetros de los retardos de la variable dependiente tiene signo negativo. Como vimos, sin embargo, existe algo de sobre-dispersión, probable consecuencia de variables omitidas $y / 0$ de conductas sistemáticas no totalmente controladas ${ }^{13}$.

\section{AGRADECIMIENTOS}

Agradecemos los comentarios de Ferran Ballester, Santiago Pérez-Hoyos y Juan Bellido. Estamos igualmente agradecidos a Carme Borrell y a Rocío Maldonado, del «Institut Municipal de la Salut», Barcelona; y a Esther Farrés de la «Unitat Ambiental» de la «Corporació Metropolitana de Barcelona», por los datos suministrados.

\section{BIBLIOGRAFÍA}

1. Ballester Díez F, Terñas JM y Pérez-Hoyos S. Efectos de la contaminación atmosférica sobre la salud: una introducción. Rev Esp Salud Pública 1999; 73:109-121.

2. Touloumi G, Katsouyanni K, Zmirou D, Schwartz J, Spix C, Ponce de Leon, A, Tobias A, Quennel P, Rabczenko D, Bacharova L, Bisanti L, Vonk JM, Ponka A. Short-term effects of ambient oxidant exposure on mortality: A combined analysis within the APHEA project. Am J Epide miol 1997(2):177-185.

3. Sunyer J, Castellsagué J, Sáez M, Tobias A, Antó JM. Air pollution and mortality in Barcelona. $J$ Epidemiol Community Health, 199650(Suppl 1): S76-S80.

4. World Health Organisation. Air quality guidelines for Europe. Copenhaguen: World Health Organisation. Regional Office for EuropeWHO. Regional Publications, European series n. 23 ; 1987.

5. Aceves M, Grimalt J. Large and small particle size screening of organic compounds in urban air. Atmosphere Environment 1993 27:251-253.

6. Sunyer J, Antó JM, Murillo C, Sáez M. Air pollution and emergency room admissions for chronic obstructive pulmonary diseases. Am J Epidemiol 1991:277-286.

7. Kinney PL, Ozkaynak H. Associations of daily mortality and air pollution in Los Angeles County. Environmental Research 1991:99-120.

8. Wietlisbach V, Pope CA, Ackermann-Liebrich U. Air pollution and daily mortality in three Swiss urban areas. Soc Prev Med 1996:107-115.

9. Verhoeff AP, Hoek G, Schwartz J. et al. Air pollution and daily mortality in Amsterdam, the Netherlands. Epidemiol 1996:225-230.

10. Ostro B, Sánchez JM, Aranda C. et al. Air pollution and mortality: results from a study of Santiago, Chile. J Exposure An Environ Epidemiol 1996:97-114.

11. Sunyer I, Sáez M, Murillo C, Castellsagué J, Martinez $\mathrm{F}$, Antó JM. Air pollution and emergency room admissions for chronic obstructive pulmonary diseases. Am J Epidemiol 1993:701-705.

12. Castellsagué J, Sunyer J, Sáez M, Antó JM. Short-term association of urban air pollution with emergency room visits for asthma. Thorax, 50:1051-1056, 1995.

13. Sáez M, Pérez-Hoyos S, Tobías A, Saurina C, Barceló M. A y Ballester F. Métodos de series temporales en los estudios epidemiológicos sobre contaminación atmosférica. Rev Esp Salud Pública $1999 ; 73: 133-143$.

14. Pérez-Hoyos S, Sáez Zafra Marc, Barceló M. ${ }^{a} A$, Cambra C, Figueiras Guzmán A, Ordoñez JM. ${ }^{a}$ et al. Protocolo EMECAM. Análisis del efecto a corto plazo de la contaminación atmosférica sobre la mortalidad. Rev Esp Salud Pública 1999; 73: $175-185$.

15. Touloumi G. Health effects of air pollution and meteorologic variables: methodological considerations with reference to the Athens case. [Tesis Doctoral]. University of Athens Medical School, Atenas, Grecia, 1994. 